(APRI) score, Fibrosis (FIB)-4 score, P3NP and HA. Statistical analysis was performed using Stata MP 15.1.

Results 3600 TEs were eligible for analysis. 104 SPs (2.9\%) had TE $\geq 7.5 \mathrm{kPa}$. 8 SPs with suspected NAFLD had TE $\geq$ $11.7 \mathrm{kPa}, 1 \mathrm{SP}$ with suspected ARLD had TE $\geq 12.5 \mathrm{kPa}$. $472(13.1 \%)$ SPs gave a history of excessive daily alcohol consumption or AUD. Of this group, 5.7\% had TE $\geq 7.5 \mathrm{kPa}$. No non-invasive scores or scores accurately identified $\geq \mathrm{F} 2$ equivalent TE $(\geq 7.5 \mathrm{kPa})$ in all SPs (area under ROC (AUROC) range $0.37-0.65$ ). These results were reflected when assessing AUROCs to detect $\geq F 2$ equivalent fibrosis $(\geq 7.9$ $\mathrm{kPa}$ ) in SPs with suspected NAFLD (AUROC range 0.390.64). In SPs with suspected ARLD, APRI had a modest diagnostic accuracy (AUROC 0.73); all other markers and scores performed poorly in diagnosing $\geq \mathrm{F} 2$ equivalent fibrosis (AUROC range $0.24-0.67$, excluding APRI).

Conclusions Non-invasive fibrosis scores have limited corroboration with TE in diagnosing moderate ARLD or NAFLD fibrosis. A study limitation is fibrosis scores were not compared to gold standard liver biopsy, in the setting of a large population study of young healthy adults; results should be interpreted in this context. The Enhanced Liver Fibrosis (ELF) test was not analysed, however, P3NP and HA - 2 of the ELF constituents - had low AUROCs.

\section{PTU-002 DENDRITIC CELL SUBSETS AND SERUM INTERLEUKIN-12 ARE LINKED TO RENAL INJURY IN SCHISTOSOMAL HEPATIC FIBROSIS}

${ }^{1}$ Hayam El Aggan*, ${ }^{2}$ Mona Salem, ${ }^{3}$ Wessam El Gendy, ${ }^{4}$ Wageh El Gebaly, ${ }^{1}$ Eman El Gohary. 'Department of Medicine, Faculty of Medicine, University of Alexandria, Alexandria, Egypt; ${ }^{2}$ Department of Pathology, Faculty of Medicine, University of Alexandria, Alexandria, Egypt; ${ }^{3}$ Department of Clinical Pathology, Faculty of Medicine, University of Alexandria, Alexandria, Egypt; ${ }^{4}$ Department of Parasitology, Faculty of Medicine, University of Alexandria, Alexandria, Egypt

\subsection{6/gutjnl-2019-BSGAbstracts.211}

Introduction Chronic infection with schistosomiasis is associated with down-regulation of $\mathrm{T}$-cell immune responses that require activation by innate immune cells like dendritic cells (DCs). The present work was designed to study the DC subsets (myeloid and plasmacytoid) in peripheral blood and serum levels of interleukin (IL)-12 in patients with schistosomal hepatic fibrosis (SHF) in relation to severity of liver disease and renal injury.

Methods Forty five patients with SHF and 15 healthy subjects were included in the study. The severity of liver disease was assessed using Child-Pugh classification and the Model of End Stage Liver Disease (MELD) score. Renal injury was assessed by measuring urinary albumin excretory rate (UAER) and estimated glomerular filtration rate (eGFR). The percentages of DC subsets in peripheral blood were detected using 3-color flow cytometric assay. Dendritic cells were identified as lineage marker negative (lin-)/HLA-DR positive cells and the differentiation of myeloid DC subset from lymphoid DC subset was based on the expression of CD11c or CD123 on the cell surface respectively. Quantitative determination of IL-12p70 heterodimer in serum was performed using enzyme-linked immunosorbent assay. Renal biopsies from patients with macroalbuminuria were examined by immunohistochemical technique for DCs and angiogenesis using antibodies against OX62 and factor VIII-related antigen respectively.
Results The percentages of circulating $\mathrm{CD} 11 \mathrm{c}^{+}$and $\mathrm{CD} 123^{+}$ DC cells, CD $11 c^{+}$DC/CD $123^{+}$DC ratio and serum IL-12 levels showed significant decreases in patients with micro- and macro-albuminuria compared with patients with normo-albuminuria and healthy subjects $(P<0.01)$. Renal tissues from patients with macroalbuminuria showed significant increases in the number of DCs and angiogenesis compared with normal renal tissues $(P<0.01)$. The percentages of circulating DC subsets and serum IL-12 levels were inversely correlated with Child-Pugh score, MELD score, number of renal DCs, renal angiogenesis and UAER and were positively correlated with eGFR. The number of renal DCs showed positive correlations with UAER and renal angiogenesis and negative correlation with eGFR $(P<0.05)$.

Conclusions DCs and IL12 seems to play a role in the progression of liver disease and renal injury in SHF. DC-based vaccines may provide a potential new goal for immunotherapy in SHF.

\section{PTU-003 MICRORNA-17 HOST GENE PROTEIN AS A POTENTIAL BIOMARKER FOR HEPATIC FIBROSIS IN CHRONIC HCV INFECTION}

${ }^{1}$ Hoda El Aggan*, ${ }^{2}$ Sabah Mahmoud, ${ }^{3}$ Nevine El Deeb, 'Ehab Hassona, 'Sally El Demiry. ${ }^{1}$ Department of Medicine (Hepatobiliary Unit), Faculty of Medicine, University of Alexandria, Alexandria, Egypt; ${ }^{2}$ Department of Medical Biochemistry, Faculty of Medicine, University of Alexandria, Alexandria, Egypt; ${ }^{3}$ Department of Pathology, Faculty of Medicine, University of Alexandria, Alexandria, Egypt

\subsection{6/gutjnl-2019-BSGAbstracts.212}

Introduction MicroRNAs (miRs) are recognized as major players in various biological processes. Circulating miRs can be used as non-invasive, sensitive biomarkers for detecting disease. The polycistronic miR-17 92 cluster is comprised of six miRs and its primary transcript also encodes for a polypeptide of 70 amino acids designated as the miR-17 host gene (MIR17HG) protein. The present study was designed to evaluate plasma MIR17HG protein as a potential biomarker for hepatic fibrosis in patients with chronic hepatitis $\mathrm{C}$ virus (HCV) infection.

Methods Thirty treatment-naïve patients with chronic HCV infection [18 patients with chronic hepatitis $\mathrm{C}(\mathrm{CHC})$ and 12 patients with cirrhosis] and 15 healthy subjects were included in the study. Quantitative determination of plasma levels of MIR17HG protein was performed using sandwich enzyme immunoassay. Core liver biopsies were obtained from all patients and were assessed for METAVIR histological activity grade and fibrosis stage as well as steatosis grade. Fibrosis scores including aspartate aminotransferase to platelet ratio index (APRI) and Fibrosis-4 (FIB-4) score were calculated. Receiver operating characteristic (ROC) curve was plotted to assess the performance of plasma MIR17HG protein, APRI and FIB-4 score in discriminating patients with early hepatic fibrosis $(\leq F 2)$ from patients with advanced fibrosis ( $>$ F2).

Results Plasma MIR17HG protein levels were significantly higher in patients with chronic HCV infection than in healthy subjects, in patients with cirrhosis than in patients with $\mathrm{CHC}$ and in patients with advanced fibrosis than in patients with early fibrosis $(P<0.001)$. Plasma MIR17HG protein levels were positively correlated with serum levels of aminotransferases $(P=0.014$ and $P=0.036$ respectively), histological activity grade $(P=0.002)$, fibrosis stage $(P<0.001)$ and 